\title{
Three-mode two-boson Jaynes-Cummings model in trapped ions
}

\author{
Benedetto Militello ${ }^{1}$, Alexander Galkin ${ }^{2}$, Anatoly Nikitin ${ }^{2}$ \\ and Antonino Messina ${ }^{1}$ \\ ${ }^{1}$ MIUR and Dipartimento di Scienze Fisiche ed Astronomiche dell’Università di Palermo, \\ Via Archirafi, 36-I-90123 Palermo, Italy \\ 2 Institute of Mathematics, National Academy of Science of Ukraine, 3 Tereshchenkiv's ka \\ Street, 01601, Kyiv-4, Ukraine
}

Received 13 October 2006, in final form 18 November 2006

Published 20 December 2006

Online at stacks.iop.org/JPhysA/40/533

\begin{abstract}
In this paper, we analyse a two-boson three-mode Jaynes-Cummings model which can be implemented in the context of trapped ions. The symmetries of the Hamiltonian are brought to light and analysed in detail in order to solve the eigenvalue problem. The calculation of the time evolution operator shows the possibility of realizing interesting applications, such as the generation of nonclassical states.
\end{abstract}

PACS numbers: $39.10 .+\mathrm{j}, 11.30 . \mathrm{Pb}$

\section{Introduction}

The Jaynes-Cummings (JC) model [1] provides a very fruitful description of the light-matter interaction, wherein the matter is usually modelled as a two-level (pseudo-spin) system coupled to a harmonic oscillator which describes one mode of the quantized radiation. The same model is used in many other physical contexts each time one deals with a spin-like system interacting with a bosonic one. For instance, suitably tuning a cavity, it is possible to make a mode of the electromagnetic (e.m.) field resonant with a specific Bohr frequency of the atom. In this way, the interaction between a harmonic oscillator (the cavity field mode) and an effective two-level system [2] is practically realized, according to which each atomic population inversion is accompanied by the absorption or emission of one photon. Exactly this kind of processes is taken into account in the JC model.

In the context of trapped ions, JC-like Hamiltonian models are used to describe the coupling between the internal and translational degrees of freedom of a confined particle. More in detail, since in a Paul e.m. trap an ion can be confined in a 'quadratic well', its motion degrees of freedom are described as modes of a three-dimensional harmonic oscillator [3]. The action of suitable laser fields on the ion is responsible for the interaction between the translational and internal degrees of freedom which makes it possible to implement a wide 
variety of Hamiltonian models [4,5]. The flexibility of this kind of systems has been exploited for the realization of seminal experiments such as the generation of Schrödinger Cat states [6], quantum logic gates [7] and quantum teleportation [8]. The action of suitably tuned and polarized laser fields produces interactions which have the same structure of a JC model or its generalizations to multi-modal and multi-photon situations $[5,9]$.

In this paper, we investigate a special case of multi-mode multi-boson JC model which can be implemented in the context of trapped ions. The analysis of a two-dimensional two-phonon JC model has brought to light very interesting features both in the context of trapped ions [10] and in the CQED counterpart [11]. Inspired by these results, we shall focus our attention on the three-mode two-boson JC model which possesses special symmetries that render the Hamiltonian manageable and solvable. The knowledge of the eigenstates and eigenvalues of such a Hamiltonian allows us to evaluate the time evolution of the system and to discuss the possibility of realizing interesting applications aimed at generating nonclassical states such as GHZ states and W-states [12].

The paper is organized as follows. In the next section, we introduce the Hamiltonian we shall analyse, while in section 3 we discuss the symmetries of such an interaction model which make it possible to find eigenfunctions of the Hamiltonian operator. In section 4, the time evolution of the system with some special initial conditions is considered and the possibility of generating of interesting nonclassical states is discussed. Finally, some conclusive remarks are given in section 5 .

\section{Hamiltonian model: implementation in trapped ions}

The electromagnetic Paul trap generates a time-dependent inhomogeneous (in particular quadrupole) electromagnetic (e.m.) field that induces the charged particle motion including two contributions, i.e., a 'secular motion' and a 'micromotion' [3]. The first one is the motion of a particle confined in a three-dimensional atomic well. The second one is a small contribution produced by rapidly oscillating terms, so that it can be neglected when a coarsegrained dynamics is considered [4]. The net result is that the behaviour of a charged particle inside an e.m. Paul trap is well approximated by the three-dimensional harmonic oscillator. When the confined particle is an ion, it is characterized by both translational and internal degrees of freedom, connected with the motion of the electrons with respect to the nucleus. In most of the practical applications, only two atomic levels of confined ions are effectively involved in the dynamics. Moreover, suitably adjusting the e.m. fields in the trap, it is possible to render the three frequencies of oscillations of the ion centre of mass to be equal and obtain a degenerate trap [13]. Therefore, the system may be 'effectively' described by the following 'free' Hamiltonian:

$$
\hat{H}_{0}=\frac{\hbar \omega_{A}}{2} \hat{\sigma}_{3}+\hbar \omega_{T} \sum_{j=x, y, z} \hat{a}_{j}^{\dagger} \hat{a}_{j},
$$

where $\omega_{A}$ is the two-level ion Bohr frequency, $\omega_{T}$ is the frequency of the degenerate trap whose annihilation (creation) operators are $\hat{a}_{j}\left(\hat{a}_{j}^{\dagger}\right), \hat{\sigma}_{3}$ is the diagonal Pauli matrix expressible as $\hat{\sigma}_{3}=|+\rangle\langle+|-|-\rangle\langle-|$, with $|+\rangle$ and $|-\rangle$ the excited and ground states of the ion, respectively.

The energy levels and eigenvectors of $\hat{H}_{0}$ are, respectively, given by

$$
\begin{aligned}
& \frac{E}{\hbar}=\omega=\omega_{A}+\left(m_{x}+m_{y}+m_{z}\right) \omega_{T} \\
& \left|m_{x}\right\rangle_{x}\left|m_{y}\right\rangle_{y}\left|m_{z}\right\rangle_{z}| \pm\rangle \equiv\left|m_{x}\right\rangle_{x} \otimes\left|m_{y}\right\rangle_{y} \otimes\left|m_{z}\right\rangle_{z} \otimes| \pm\rangle .
\end{aligned}
$$


Considering a one-dimensional centre-of-mass motion, the frequency $\omega=\omega_{A}+k \omega_{T}$ is referred to as the carrier, the $k$ th red sideband or the $k$ th blue sideband depending on being $k=0, k<0$ or $k>0$, respectively.

One of the most interesting virtues of trapped ions is the possibility of implementing Hamiltonian models practically at will. Acting upon the ion with a suitably tuned and polarized laser field it is possible to obtain nonlinear $k$-phonon Jaynes-Cummings models. The action of more than one laser is described via the sum of the Hamiltonian models related to each laser fields. For example, applying a laser field propagating along the $x$-direction and tuned to the red blue sideband, $\omega=\omega_{A}-\omega_{T}$, we induce transitions characterized by atomic population inversions accompanied by the absorption/emission of one boson of the vibrational 'mode' associated with the motion along $x$. In other words, the interaction has the structure of a standard JC interaction term associated with the mode related to $\hat{a}_{x}$. The simultaneous application of three laser fields tuned to the second red sideband, directed along three orthogonal directions, $x, y$ and $z$, and with suitable intensities, generates the following interaction picture Hamiltonian model (for details of the derivation, see appendix A):

$$
\hat{H}_{\text {int }}=\hbar \Omega\left(\hat{a}_{x}^{\dagger 2}+\hat{a}_{y}^{\dagger 2}+\hat{a}_{z}^{\dagger 2}\right) \hat{\sigma}_{-}+\text {h.c. },
$$

where $\Omega$ is the coupling strength and $\hat{\sigma}_{ \pm}:=| \pm\rangle\langle\mp|$ are the Pauli ladder operators.

\section{Properties of the Hamiltonian}

\subsection{Symmetries}

The interaction picture Hamiltonian in (A.7) is a product of bosonic terms such as $\sum_{j=x, y, z} \hat{a}_{x}^{\dagger 2}$ and $\sum_{j=x, y, z} \hat{a}_{x}^{2}$ which are multiplied to the spin operators $\hat{\sigma}_{ \pm}$. Each of these bosonic parts possesses a $s o(3)$ symmetry, being invariant under rotations around all axes.

This circumstance reflects on the fact that the orbital angular momentum $\vec{L}$ of the oscillator, defined as follows

$$
\left\{\begin{array}{l}
\vec{L}:=\left(\hat{L}_{x}, \hat{L}_{y}, \hat{L}_{z}\right) \\
\hat{L}_{k}=\mathrm{i}\left(\hat{a}_{j}^{\dagger} \hat{a}_{l}-\hat{a}_{l}^{\dagger} \hat{a}_{j}\right) \quad j, l, k=x, y, z \text { and cyclic, }
\end{array}\right.
$$

is a constant of motion. As usual, in order to obtain a complete set of commuting operators (CSCO), we shall consider two commuting operators, i.e., the square of the angular momentum, $\hat{L}^{2}:=\hat{L}_{x}^{2}+\hat{L}_{y}^{2}+\hat{L}_{z}^{2}$, and the third component $\hat{L}_{z}$.

The total excitation number operator,

$$
\hat{N}=\sum_{j=x, y, z} \hat{a}_{j}^{\dagger} \hat{a}_{j}+\hat{\sigma}_{3}+1 \equiv \hat{N}_{0}+\hat{\sigma}_{3}+1
$$

that includes the fermionic excitation number $\hat{\sigma}_{3}$ as well as the total bosonic excitation number operator $\hat{N}_{0}$, is a constant of motion. We mention that such conservation law is connected with the invariance of the Hamiltonian under the following canonical transformation [11]:

$$
\hat{a}_{j} \rightarrow \mathrm{e}^{\mathrm{i} \phi} \hat{a}_{j}, \quad \hat{\sigma}_{ \pm} \rightarrow \mathrm{e}^{\mp \mathrm{i} 2 \phi} \hat{\sigma}_{ \pm} .
$$

The operators

$$
\hat{N}, \hat{L}^{2}, \hat{L}_{z}
$$

all commute each other and with both $\hat{H}_{0}$ and $\hat{H}_{\text {int }}$, and hence may be used to find the Hamiltonian eigenfunctions. It is worth noting that such three operators also commute with the unperturbed Hamiltonian $\hat{H}_{0}$, so that they really are constants of motion, in the sense that no explicit time dependence has been introduced in the passage to the interaction 
picture. It follows immediately from (5) that the eigenstates of $\hat{N}_{0}$ timed the eigenstates of $\hat{\sigma}_{3}$ are eigenfunctions of $\hat{N}$. Moreover, eigenfunctions of the three operators $\hat{N}_{0}, \hat{L}^{2}$ and $\hat{L}_{z}$ timed the eigenstates of the spin operator $\hat{\sigma}_{3}$ are simultaneous eigenstates of the three operators in (7) and $\hat{\sigma}_{3}$, and hence form a suitable basis for expanding the solutions of the Hamiltonian eigenvalue problem. We will denote the related bases vectors as $|\Phi(n, l, m, \sigma= \pm)\rangle:=\left|\Psi\left(n_{0}=n \mp 1-1, l, m\right)\right\rangle \otimes|\sigma= \pm\rangle$; eigenstates of $\hat{N}, \hat{L}^{2}, \hat{L}_{z}$ and $\hat{\sigma}_{3}$ on the left-hand side and eigenstates of $\hat{N}_{0}, \hat{L}^{2}, \hat{L}_{z}$ timed eigenstates of $\hat{\sigma}_{3}$ on the right-hand side.

\subsection{Solving the eigenvalue problem}

Because of the commutation between the interaction picture Hamiltonian and the 'conserved operators' in (7), each couple of states $|\Phi(n, l, m, \sigma= \pm)\rangle$ (which are also eigenstates of $\hat{H}_{0}$ ) constitutes an invariant subspace under the action of the Hamiltonian $\hat{H}_{\text {int }}$. Therefore, the Hamiltonian problem can be solved algebraically diagonalizing each $2 \times 2$ block. Alternatively, it is possible to find a unitary transformation that realizes such a diagonalization. Indeed, due to the special structure of the Hamiltonian, a Foldy-Wouthuysen-type transformation (FWT) $[14,15]$ can be exploited to this end:

$$
\hat{U} \equiv \exp \left(-\frac{\pi}{4} \frac{\hat{Q}^{\dagger}-\hat{Q}}{\sqrt{\hat{H}_{\mathrm{int}}^{2}}}\right),
$$

with

$$
\begin{aligned}
& \hat{Q}=\hbar \Omega\left[\sum_{j=x, y, z} \hat{a}_{x}^{\dagger 2} \hat{\sigma}_{-},\right. \\
& \hat{H}_{\text {int }}^{2}=(\hbar \Omega)^{2}\left[\hat{N}^{2}+\hat{N}-\hat{L}^{2}\right],
\end{aligned}
$$

which leads to

$$
\hat{H}_{\text {int }}^{\text {diag }} \equiv \hat{U}^{\dagger} \hat{H}_{\text {int }} \hat{U}=\sqrt{\hat{H}_{\text {int }}^{2}} \hat{\sigma}_{3} .
$$

Once the canonical transformation in (11) has been realized, the diagonalization may be completed finding the common eigensolutions of the total excitation number $\hat{N}$, the angular momentum $\hat{L}^{2}$ and of the third Pauli matrix $\hat{\sigma}_{3}$ (the structure of the spectrum is given in appendix B, equation (B.2)). To obtain a complete set of commuting operators (CSCO) we add the third component $\hat{L}_{z}$ of the angular momentum operator. For a fixed value of $\hat{\sigma}_{3}$ we have to find the common eigenstates of $\hat{N}_{0}:=\sum_{j=x, y, z} \hat{a}_{j}^{\dagger} \hat{a}_{j}, \hat{L}^{2}$ and $\hat{L}_{z}$, here denoted by $\left|\Psi\left(n_{0}, l, m\right)\right\rangle$, which include only translational degrees of freedom. Such a well-known problem may be straightforwardly solved [20] considering the action of the lowering and raising angular momentum operators $\hat{L}_{ \pm}$. Passing from the Fock basis to that under scrutiny may be performed with the help of (C.15) reported in the appendix and derived for instance in [22].

In accordance with (5), it is convenient to make the following identification of common states of $\hat{N}, \hat{L}^{2}, \hat{L}_{z}, \hat{\sigma}_{3}$ (left-hand side) and $\hat{N}_{0}, \hat{L}^{2}, \hat{L}_{z}, \hat{\sigma}_{3}$ (right-hand side):

$$
|\Phi(n, l, m, \sigma= \pm)\rangle \equiv\left|\Psi\left(n_{0}=n-1 \mp 1, l, m\right)\right\rangle \otimes|\sigma= \pm\rangle .
$$

It is easy to find the basis $\left|n, l, m, e_{n, l}\right\rangle$ of common eigenstates of the CSCO $\hat{N}, \hat{L}^{2}, \hat{L}_{z}, \hat{H}_{\text {int }}$ using the inverse FWT:

$$
\left|n, l, m, e_{n, l}\right\rangle=\hat{U}|\Phi(n, l, m, \sigma)\rangle .
$$


Since the generator of $\hat{U}$ commutes with $\hat{N}, \hat{L}^{2}$ and $\hat{L}_{z}$, the effect of the transformation is restricted to each $\left(\hat{N}, \hat{L}^{2}, \hat{L}_{z}\right)$-invariant subspace and simply realizes a mixing of the states $|\Phi(n, l, m,+)\rangle$ and $|\Phi(n, l, m,-)\rangle$.

As an example we expand some angular momentum basis states in terms of the Fock basis:

$$
\begin{aligned}
& |\Psi(0,0,0)\rangle=|0\rangle_{x}|0\rangle_{y}|0\rangle_{z} \\
& |\Psi(2,0,0)\rangle=\frac{1}{\sqrt{3}}|2\rangle_{x}|0\rangle_{y}|0\rangle_{z}+\frac{1}{\sqrt{3}}|0\rangle_{x}|2\rangle_{y}|0\rangle_{z}+\frac{1}{\sqrt{3}}|0\rangle_{x}|0\rangle_{y}|2\rangle_{z} \\
& |\Psi(2,2,0)\rangle=\frac{1}{\sqrt{6}}|2\rangle_{x}|0\rangle_{y}|0\rangle_{z}+\frac{1}{\sqrt{6}}|0\rangle_{x}|2\rangle_{y}|0\rangle_{z}-\sqrt{\frac{2}{3}}|0\rangle_{x}|0\rangle_{y}|2\rangle_{z}
\end{aligned}
$$

from which it follows, for example,

$$
\begin{aligned}
|2,0,0, \pm \hbar \Omega \sqrt{6}\rangle=\frac{1}{\sqrt{6}}\left[|2\rangle_{x}|0\rangle_{y}|0\rangle_{z}|-\rangle\right. \\
\left.\quad+|0\rangle_{x}|2\rangle_{y}|0\rangle_{z}|-\rangle+|0\rangle_{x}|0\rangle_{y}|2\rangle_{z}|-\rangle\right] \pm \frac{1}{\sqrt{2}}|0\rangle_{x}|0\rangle_{y}|0\rangle_{z}|+\rangle
\end{aligned}
$$

Analogous results may be systematically found for all other subspaces.

\section{Time evolutions}

On the basis of the previous calculation, it is straightforward to evaluate the time evolution of the state

$$
|\psi(t=0)\rangle=|0\rangle_{x}|0\rangle_{y}|0\rangle_{z}|+\rangle .
$$

Such a state may be easily generated in trapped ion systems. Indeed, once the system has been lead to the vibronic ground state $|0\rangle_{x}|0\rangle_{y}|0\rangle_{z}|-\rangle$ through cooling techniques $[4,16]$, it is enough to induce an atomic population inversion. The relevant time evolution can be expressed as

$$
\begin{aligned}
|\psi(t)\rangle= & \cos (\sqrt{6} \Omega t)|0\rangle_{x}|0\rangle_{y}|0\rangle_{z}|+\rangle \\
& -\frac{\mathrm{i}}{\sqrt{3}} \sin (\sqrt{6} \Omega t)\left[|2\rangle_{x}|0\rangle_{y}|0\rangle_{z}|-\rangle+|0\rangle_{x}|2\rangle_{y}|0\rangle_{z}|-\rangle+|0\rangle_{x}|0\rangle_{y}|2\rangle_{z}|-\rangle\right] .
\end{aligned}
$$

After a $\frac{\pi}{2}$-pulse, i.e. at the instant of time $t_{0}$ such that $\sqrt{6} \gamma t_{0}=\frac{\pi}{2}$, up to a global phase factor, the system is found to be in the GHZ state [12]:

$$
\left|\psi\left(t_{0}\right)\right\rangle=\frac{1}{\sqrt{3}}|2\rangle_{x}|0\rangle_{y}|0\rangle_{z}|-\rangle+|0\rangle_{x}|2\rangle_{y}|0\rangle_{z}|-\rangle+|0\rangle_{x}|0\rangle_{y}|2\rangle_{z}|-\rangle .
$$

The importance of such a state relies in its applications in the study of the Bell's inequality violations. In passing, we observe that, as expected, starting from the spherically symmetric wavefunction given in (15), the dynamics preserves such a symmetry at any instant of time.

As another example consider a non-spherically symmetric initial state which distinguishes the $z$-direction from the other two. Let

$$
|\psi(t=0)\rangle=|0\rangle_{x}|0\rangle_{y}|2\rangle_{z}|-\rangle .
$$

The relevant time evolution is then given by

$$
\begin{aligned}
|\psi(t)\rangle=\frac{1}{3}[\cos (\sqrt{6} \Omega t)+2]|0\rangle_{x}|0\rangle_{y}|2\rangle_{z}|-\rangle \\
\quad+\frac{1}{3}[\cos (\sqrt{6} \Omega t)-1]\left[|2\rangle_{x}|0\rangle_{y}|0\rangle_{z}|-\rangle+|0\rangle_{x}|2\rangle_{y}|0\rangle_{z}|-\rangle\right] \\
\quad-\frac{\mathrm{i}}{\sqrt{3}} \sin (\sqrt{6} \Omega t)|0\rangle_{x}|0\rangle_{y}|0\rangle_{z}|+\rangle .
\end{aligned}
$$


Due to the fact that $\left\{|2\rangle_{x}|0\rangle_{y}|0\rangle_{z}|-\rangle,|0\rangle_{x}|2\rangle_{y}|0\rangle_{z}|-\rangle,|0\rangle_{x}|0\rangle_{y}|2\rangle_{z}|-\rangle,|0\rangle_{x}|0\rangle_{y}|0\rangle_{z}|+\rangle\right\}$ generates an excitation number invariant subspace, the states involved in the two considered dynamics, the ones in equations (16) and (19), are the same. Nevertheless, the weights are significantly different. In particular, in the second case, no instant of time exists when the state $|\psi(t)\rangle$ coincides with $|0\rangle_{x}|0\rangle_{y}|0\rangle_{z}|+\rangle$.

\section{Conclusive remarks}

In this paper, we have described a physical scenario wherein a three-dimensional two-phonon Jaynes-Cummings-like Hamiltonian model is implementable and, thanks to its symmetries, solvable. Interesting dynamical features have been brought to light. A confined ion into a Paul trap, subjected to a suitable laser field configuration, is describable by an interaction picture vibronic coupling quadratic in the annihilation (creation) bosonic operators. Such a Hamiltonian possesses symmetries connected to the excitation number conservation, the rotational invariance (i.e. conservation of angular momentum) and a hidden second derivative supersymmetry which may be used to individualize a suitable diagonalizing FoldyWouthuysen-like transformation, see appendix B for more detail.

We use the FWT together with the representation of the interaction Hamiltonian in terms of generators of $s u(1,1)$ algebra to obtain exact solutions of the eigenvalue problem (refer to appendix $\mathrm{C}$ ). The procedure we propose appears to be remarkably simple and straightforward and could be generalized to more complicated systems.

It is worth remarking that the success of our approach comes from the particular features of the Hamiltonian we have considered, and especially from the fact that it possesses a so(3) symmetry coming from the presence of only squares of the annihilation/creation operators in the interaction Hamiltonian. In fact, in the multi-boson Jaynes-Cummings model involving a number of bosons different from 2 these symmetries are seemingly lost and the model turns out to be more difficult to solve [21]. Another important point is the role of the trap isotropy which contributes to the simple structure of the Hamiltonian. Concerning this point, it is worth remarking that in the two-dimensional case it has been shown that the analogous of our model in the absence of isotropy exhibits a richer dynamics than in the presence of trapfrequency complete degeneration. Nevertheless such conclusions, reported in [23], pertain to a class of states quite different from the ones here analysed. In addition, the extension of the Hamiltonian-digonalization procedure from the two-dimensional to the three-dimensional anisotropic model is seemingly not straightforward. Therefore, the generalization of the role of anisotropy from the 2D case to the $3 \mathrm{D}$ context is not trivial and goes beyond the scope of this paper.

Our main result is the proof of the possibility of generating nonclassical states such as GHZ-like state involving the three phononic centre-of-mass modes. This possibility relies on the fact that the dynamical behaviour of the system preserves the symmetries of the initial states. Thus, for example, starting from a completely spherically symmetric state possessing two total excitations but no vibrational excitations (see (15)), we forecast the generation of a state possessing three vibrational excitations equally distributed among the three bosonic modes, as in (17). In contrast, in (19), no instant of time exists at which it is possible to obtain a spherically symmetric state. Indeed, the initial state distinguishes the $z$ axis from the other two, in the sense that the excitations are not equally distributed through the three axes, and such an inhomogeneity is kept at any further instant of time. 


\section{Appendix A. Physical implementation of the Hamiltonian}

In this appendix, we deduce the Hamiltonian model in (3). Let us first of all analyse the case when a single laser is turned on, so that the relevant interaction Hamiltonian may thought of as a one-dimensional operator. In this situation, the Schrödinger picture interaction Hamiltonian may be written as

$$
\hat{H}_{1 D}^{(S)}=-\vec{d} \cdot \vec{E}(\vec{r}, t)
$$

where $\vec{d}=\vec{d}_{ \pm} \hat{\sigma}_{+}+\vec{d}_{ \pm}^{*} \hat{\sigma}_{-}$is the two-level restriction of the atomic dipole operator and $\vec{E}(\vec{r}, t)$ is the electric field in the centre of mass of the ion $\vec{r} \equiv(\hat{X}, \hat{Y}, \hat{Z})$. Assuming the propagation in the $x$-direction one has

$$
\begin{aligned}
\vec{E}(\vec{r}, t) & \equiv \vec{E}_{0} \mathrm{e}^{\mathrm{i}\left(\vec{k} \cdot \vec{r}-\omega_{L} t\right)}+\vec{E}_{0}^{*} \mathrm{e}^{-\mathrm{i}\left(\vec{k} \cdot \vec{r}-\omega_{L} t\right)} \\
& =\vec{E}_{0} \mathrm{e}^{\mathrm{i}\left(k_{x} \hat{X}-\omega_{L} t\right)}+\vec{E}_{0}^{*} \mathrm{e}^{-\mathrm{i}\left(k_{x} \hat{X}-\omega_{L} t\right)}
\end{aligned}
$$

Passing to the interaction picture and expanding the exponentials with the help of the Baker-Campbell-Hausdorff formula, one obtains the following time-dependent interaction:

$\hat{H}_{1 D}=\hbar g_{x}\left[\mathrm{e}^{-\frac{\eta_{L}^{2}}{2}} \sum_{s, j=0}^{\infty} \frac{\left(-\mathrm{i} \eta_{x}\right)^{s+j}}{s ! j !}\left(\hat{a}_{x}^{\dagger}\right)^{j} \hat{a}_{x}^{s} \mathrm{e}^{\mathrm{i}(j-s) \omega_{T} t} \mathrm{e}^{-\mathrm{i}\left(\omega_{A}-\omega_{L}\right) t}\right] \hat{\sigma}_{-}+$h.c.

where $g_{x} \equiv \vec{d}_{ \pm} \cdot \vec{E}_{0, x} \eta_{x} \equiv \frac{k_{x}}{2 \pi} \sqrt{\frac{\hbar}{\mu \omega_{T}}}$, with $\mu$ being the mass of the ion is the so-called Lamb-Dicke parameter $\eta_{x}$ expressing the ratio between the amplitude of the oscillations of the ion centre of mass in the bosonic ground state to the laser field wavelength. Therefore, such a parameter governs the influence of the electromagnetic field gradient to the laser-driven trapped ion dynamics. Tuning the laser field to the $m$ th red sideband, i.e. in such a way that

$$
\omega_{L}=\omega_{A}-m \omega_{T},
$$

it results that the term corresponding to $j$ and $s$ oscillates due the phase factor $\mathrm{e}^{(j-s-m) \omega_{T} t}$. Hence, the only stationary interaction terms are those for which $j-s-m=0$. Under the rotating wave approximation (RWA), i.e. discarding all the rapidly oscillating terms, one finds

$$
\hat{H}_{1 D}=\hbar g_{x} \mathrm{e}^{-\frac{\eta_{L}^{2}}{2}}\left(-\mathrm{i} \eta_{x}\right)^{m}\left(\hat{a}_{x}^{\dagger}\right)^{m} \sum_{s=0}^{\infty} \frac{\left(-\mathrm{i} \eta_{x}\right)^{2 s}}{s !(s+m) !}\left(\hat{a}_{x}^{\dagger}\right)^{s} \hat{a}_{x}^{s} \hat{\sigma}_{-}+\text {h.c.. }
$$

Assume now that the laser field wavelength is much larger than the amplitude of the oscillations of the ion centre of mass. Such an assumption reflects into the condition of very small Lamb-Dicke parameter, $\eta_{x} \ll 1$ usually referred to as Lamb-Dicke limit, under which we obtain (retaining only the zero-order terms in the series)

$$
\hat{H}_{1 D} \approx-\hbar \frac{\left(-\mathrm{i} \eta_{x}\right)^{m} g_{x}}{m !}\left(\hat{a}_{x}^{\dagger}\right)^{m} \hat{\sigma}_{-}+\text {h.c. }
$$

that is a $m$-boson Jaynes-Cummings interaction Hamiltonian model. The net result of introducing the Lamb-Dicke approximation is to get rid of the nonlinearities present in the series reported in (A.5).

Consider now the simultaneous action of three laser fields tuned to the second red sideband, directed along three orthogonal directions, $x, y$ and $z$, and in the Lamb-Dicke limit. The relevant interaction picture Hamiltonian is given by

$$
\hat{H}_{\mathrm{int}}=\hbar\left[\sum_{j=x, y, z} \frac{\eta_{j}^{2} g_{j}}{2} \hat{a}_{x}^{\dagger 2}\right] \hat{\sigma}_{-}+\text {h.c.. }
$$

Adjusting the three laser intensities in such a way that $\Omega=\frac{\eta_{j}^{2} g_{j}}{2}$ for $j=x, y, z$, the final result is the isotropic three-dimensional interaction Hamiltonian model in (3). 


\section{Appendix B. Foldy-Wouthuysen-like transformation}

In this appendix, we discuss the origin of the unitary transformation in (8). As given in (8), the square of the interaction picture Hamiltonian is

$$
\hat{H}_{\text {int }}^{2}=(\hbar \Omega)^{2}\left[\hat{N}^{2}+\hat{N}-\hat{L}^{2}\right] .
$$

All operators involved in (B.1) commute each other thus this formula can be treated as an algebraic equation for eigenvalues of $\hat{H}_{\mathrm{int}}$. Using (B.1) we can determine, up to a sign, the eigenvalues of $\hat{H}_{\text {int }}, e_{n, l}$, as

$$
\left|e_{n, l}\right|=\hbar \Omega \sqrt{[n(n+1)-l(l+1)]},
$$

with $n$ and $l$ being the positive integer quantum numbers related to the excitation number operator $(\hat{N})$ and to the square of the angular momentum $\left(\hat{L}^{2}\right)$.

In addition to the individualization of the eigenvalue structure of $\hat{H}_{\text {int }}$, equation (B.1) strongly suggests the existence of a Foldy-Wouthuysen-like transformation (FWT) able to diagonalize our Hamiltonian model $[14,15]$. Such a circumstance is related to a hidden higher derivative supersymmetry the system possesses. In order to show it let us introduce two operators $\hat{Q}$ and $\hat{Q}^{\dagger}$ :

$$
\hat{Q}=\hbar \Omega\left[\sum_{j=x, y, z} \hat{a}_{x}^{\dagger 2}\right] \hat{\sigma}_{-}, \quad \hat{Q}^{\dagger}=\hbar \Omega\left[\sum_{j=x, y, z} \hat{a}_{x}\right] \hat{\sigma}_{+} .
$$

These operators and Hamiltonian in (3) satisfy the following relations:

$$
\begin{aligned}
& \left\{\hat{Q}, \hat{Q}^{\dagger}\right\}=\hat{H}_{\text {int }}^{2}, \quad\left[\hat{H}_{\mathrm{int}}^{2}, \hat{Q}\right]=0, \\
& {\left[\hat{Q}, \hat{Q}^{\dagger}\right]=\hat{H}_{\mathrm{int}}^{2} \sigma_{3},} \\
& \left\{\hat{Q}, \sigma_{3}\right\}=\left\{\hat{Q}^{\dagger}, \sigma_{3}\right\}=0, \\
& {\left[N, \hat{H}_{\mathrm{int}}^{2}\right]=0, \quad[N, \hat{Q}]=-\hat{Q}, \quad\left[N, \hat{Q}^{\dagger}\right]=\hat{Q}^{\dagger},} \\
& {\left[\hat{H}_{\mathrm{int}}^{2}, \hat{Q}\right]=\left[\hat{H}_{\mathrm{int}}^{2}, \hat{Q}^{\dagger}\right]=0, \quad\left(\hat{Q}^{\dagger}-\hat{Q}\right)^{2}=-\hat{H}_{\mathrm{int}}^{2} .}
\end{aligned}
$$

Relations (B.4) characterize the superalgebra of SUSY quantum mechanics [17]. The even element of this algebra is represented by $\hat{H}_{\text {int }}^{2}$ whilst the interaction Hamiltonian $\hat{H}_{\text {int }}$ can be rewritten as

$$
\hat{H}_{\text {int }}=\hat{Q}^{\dagger}+\hat{Q} .
$$

Representation in (B.6) and relations in (B.4) are analogous to ones used in paper [18] to construct the FW transformation for the Dirac Hamiltonian. We note that in our case the supercharges $Q$ and $\hat{Q}$ are realized by higher (second) order differential operators.

In analogy with [18] we can use relations (B.4), (B.5) and (B.6) to write the operator of FW transformation in (8). In fact, it is easy to show that (8) holds. Observe the accordance of equation (11) with equation (B.1) due to $\hat{\sigma}_{3}^{2}=1$, keeping in mind that the unitary operator in equation (8) does not transform the square of $\hat{H}_{\text {int }}$ despite $\hat{H}_{\text {int }}$ itself is modified. Hence, squaring equation (11) gives exactly equation (B.1), which is not sensitive to the anti-transformation induced by $\hat{U}^{\dagger}$.

\section{Appendix C. Change of basis}

In this appendix, following [22], we construct the basis which belongs to the totally symmetric irreducible representation $[N, 0,0]$ of $u(3)$ algebra. In other words, we recall a general formula 
providing the expansion of angular momentum eigenstates in terms of Fock states. In the Fock space of totally symmetric irreducible representation $[N, 0,0]$ of $u(3)$, the angular momentum operators can be written in the form

$\hat{M}_{0}=b_{1}^{\dagger} b_{1}-b_{2}^{\dagger} b_{2}, \quad \hat{M}_{+}=\sqrt{2}\left[b_{1}^{\dagger} b_{3}+b_{3}^{\dagger} b_{2}\right], \quad \hat{M}_{-}=\sqrt{2}\left[b_{3}^{\dagger} b_{1}+b_{2}^{\dagger} b_{3}\right]$,

where the operators $b_{i}^{\dagger}$ and $b_{i}(i=1,2,3)$ can be expressed in terms of the operators $a_{i}^{\dagger}$ and $a_{i}$ by the following transformation:

$$
\begin{array}{lll}
b_{1}=-\frac{1}{\sqrt{2}}\left(a_{x}-i a_{y}\right), & b_{2}=-\frac{1}{\sqrt{2}}\left(a_{x}+i a_{y}\right), & b_{3}=a_{z}, \\
b_{1}^{\dagger}=-\frac{1}{\sqrt{2}}\left(a_{x}^{\dagger}+i a_{y}^{\dagger}\right), & b_{2}^{\dagger}=-\frac{1}{\sqrt{2}}\left(a_{x}^{\dagger}-i a_{y}^{\dagger}\right), & b_{3}^{\dagger}=a_{z}^{\dagger} .
\end{array}
$$

Operators in (C.1) satisfy the commutation relations of $s o(3)$ algebra:

$$
\left[\hat{M}_{0}, \hat{M}_{ \pm}\right]= \pm L_{ \pm},\left[\hat{M}_{+}, \hat{M}_{-}\right]=2 M_{0} .
$$

The normalized highest weight $\operatorname{so}(3)$ state $|l, l\rangle$ in terms of creation and annihilation operators $b_{i}$ and $b_{i}^{\dagger}(i=1,2,3)$ has the form

$$
|l, l\rangle=\frac{\left(b_{1}^{\dagger}\right)^{l}}{\sqrt{l !}}|0\rangle_{x}|0\rangle_{y}|0\rangle_{z} .
$$

Using (C.1) and commutation relations (C.3) one can verify that state $|l, l\rangle$ satisfies the conditions of highest weight state:

$$
\hat{M}_{+}|l, l\rangle=0, \quad \hat{M}_{0}|l, l\rangle=l|l, l\rangle, \quad\langle l, l \mid l, l\rangle=1 .
$$

But the state in (C.4) is not the most general highest weight $s o(3)$ state, since this state factorizes arbitrary $s o(3)$ scalar operator, which will not modify the value of $l$. These $s o(3)$ scalar operators can be expressed in the form

$$
\begin{aligned}
& \hat{S}_{+}=\frac{1}{2}\left(\left(b_{3}^{\dagger}\right)^{2}-2 b_{1}^{\dagger} b_{2}^{\dagger}\right), \\
& \hat{S}_{-}=\frac{1}{2}\left(b_{3}^{2}-2 b_{1} b_{2}\right), \\
& \hat{S}_{3}=\frac{1}{2}\left(b_{1}^{\dagger} b_{1}+b_{2}^{\dagger} b_{2}+b_{3}^{\dagger} b_{3}+\frac{3}{2}\right)=\frac{1}{2}\left(\hat{N}_{0}+\frac{3}{2}\right),
\end{aligned}
$$

where $\hat{N}_{0}$ is the total excitation number operator. These operators satisfy the commutation relations of $s u(1,1)$ algebra

$$
\left[\hat{S}_{0}, \hat{S}_{ \pm}\right]= \pm \hat{S}_{ \pm}, \quad\left[\hat{S}_{+}, \hat{S}_{-}\right]=-2 \hat{S}_{0}
$$

and commute with the generators of $s o(3)$ algebra, $\hat{M}_{ \pm}$and $\hat{M}_{0}$.

Now let us define the state which is characterized by an angular momentum $l$ and its projection $m=l$ and belong to totally symmetric irreducible representation $[N, 0,0]$ of $u(3)$ algebra as

$$
\left|\Psi\left(n_{0}, l, l\right)\right\rangle=\frac{1}{R_{n l}}\left(2 \hat{S}_{+}\right)^{\frac{1}{2}(n-l)}|l, l\rangle,
$$

where $l=n_{0}, n_{0}-2, \ldots, 0$ or 1 . The normalization constant $R_{n_{0} l}$ can be found from the condition

$$
\left\langle n_{0}, l, l \mid n_{0}, l, l\right\rangle=1,
$$

using the relations

$$
\begin{aligned}
& {\left[\hat{S}_{-}, \hat{S}_{+}^{k}\right]=2 k \hat{S}_{+}^{k}\left(2 \hat{N}_{0}+2 k+1\right),} \\
& \hat{S}_{-}|l, l\rangle=0, \quad \hat{N}_{0}|l\rangle l=l|l\rangle l,
\end{aligned}
$$


and it takes the form

$$
R_{n_{0} l}=\sqrt{\frac{(n-l) ! !(n+l+1) ! !}{(2 l+1) ! !}} .
$$

Using commutation relations in (C.6) and its consequence,

$$
\left[\hat{N}_{0}, \hat{S}_{+}^{k}\right]=2 k \hat{S}_{+}^{k},
$$

one can obtain that the state in (C.7) satisfies the following relations:

$$
\begin{aligned}
& \hat{N}_{0}\left|n_{0}, l, l\right\rangle=n_{0}\left|n_{0}, l, l\right\rangle, \quad \hat{M}_{0}\left|n_{0}, l, l\right\rangle=l\left|n_{0}, l, l\right\rangle, \\
& \hat{M}^{2}\left|n_{0}, l, l\right\rangle=l(l+1)\left|n_{0}, l, l\right\rangle,
\end{aligned}
$$

where $\hat{M}^{2}$ is the Casimir operator of $s o(3)$, which has the form

$$
\hat{M}^{2}=\hat{M}_{+} \hat{M}_{-}+\hat{M}_{0}\left(\hat{M}_{0}+1\right) .
$$

The states with an arbitrary projection of momentum $m$ can be written using the operator $\hat{M}_{-}$, i.e.,

$$
\left|\Psi\left(n_{0}, l, m\right)\right\rangle=\frac{1}{\lambda_{l m}}\left(\hat{M}_{-}\right)^{l-m}|\Psi(n, l, l)\rangle,
$$

where $\lambda_{l m}$ is the normalization constant which takes the from

$$
\lambda_{l m}=\sqrt{\frac{(2 l) !(l-m) !}{(l+m) !}} .
$$

Using equations (C.4), (C.7) and (C.9) we come to the following form of the basis vector, i.e.,

$$
\left|\Psi\left(n_{0}, l, m\right)\right\rangle=C_{n_{0} l m}\left(2 S_{+}\right)^{\frac{1}{2}\left(n_{0}-l\right)}\left(L_{-}\right)^{l-m}\left(b_{1}^{\dagger}\right)^{l}|0\rangle_{x}|0\rangle_{y}|0\rangle_{z},
$$

where the coefficient $C_{n l m}$ can be written as

$$
C_{n_{0} l m}=\sqrt{\frac{(2 l+1) ! !(l+m) !}{(2 l) ! l !(l-m) !\left(n_{0}-l\right) ! !\left(n_{0}+l+1\right) ! !}} .
$$

In order to express (C.11) in terms polynomials of the operators $b_{i}^{\dagger}(i=1,2,3)$, we use the relations

$$
\frac{\left(\hat{M}_{-}\right)^{m}\left(b_{1}^{\dagger}\right)^{l}}{\sqrt{(2 l) ! !}}|0\rangle_{x}|0\rangle_{y}|0\rangle_{z}=\sum_{p=\max (0, m)}^{(l+m) / 2} 2^{\frac{m}{2}} \frac{\left(b_{1}^{\dagger}\right)^{p}\left(b_{2}^{\dagger}\right)^{m-l+p}\left(b_{3}^{\dagger}\right)^{2 l-m-2 p}}{(2 p) ! !(2 l-m-2 p) !(2 m-2 l+2 p) ! !}|0\rangle_{x}|0\rangle_{y}|0\rangle_{z} .
$$

In this case, the basis vectors in (C.11) take the form

$$
\begin{aligned}
\left|\Psi\left(n_{0}, l, m\right)\right\rangle= & 2^{\frac{l-m}{2}} C_{n_{0} l m}\left(2 S_{+}\right)^{\frac{1}{2}\left(n_{0}-l\right)} \\
& \times \sum_{p=\max (0, m)}^{(l+m) / 2} \frac{\left(b_{1}^{\dagger}\right)^{p}\left(b_{2}^{\dagger}\right)^{p-m}\left(b_{3}^{\dagger}\right)^{l+m-2 p}}{(2 p) ! !(2 l-m-2 p) !(2 m-2 l+2 p) ! !}|0\rangle_{x}|0\rangle_{y}|0\rangle_{z} .
\end{aligned}
$$

Taking into account the binomial formula, which is true for any commuting $X$ and $Y$, we can write $\left(2 S_{+}\right)^{\frac{1}{2}\left(n_{0}-l\right)}$ in the polynomial form

$$
\left(2 \hat{S}_{+}\right)^{\frac{1}{2}\left(n_{0}-l\right)}=\sum_{s=0}^{\left(n_{0}-l\right) / 2}\left(\begin{array}{c}
s \\
\frac{1}{2}\left(n_{0}-l\right)
\end{array}\right)(-1)^{s}\left(b_{3}^{\dagger}\right)^{n_{0}-l-2 s}\left(b_{1}^{\dagger}\right)^{s}\left(b_{2}^{\dagger}\right)^{s},
$$

where $\left(\begin{array}{l}s \\ k\end{array}\right)=\frac{k !}{s !(k-s) !}$ are the binomial coefficients. 
Using (C.14), the final form of the basis vector $|\Psi(n, l, m)\rangle$ can be obtained in the polynomial form

$$
\begin{aligned}
\left|\Psi\left(n_{0}, l, m\right)\right\rangle= & 2^{\frac{l-m}{2}} C_{n_{0} l m} \sum_{s=0}^{\left(n_{0}-l\right) / 2} \sum_{p=\max (0, m)}^{(l+m) / 2}\left(\begin{array}{c}
s \\
\frac{1}{2}\left(n_{0}-l\right)
\end{array}\right)(-1)^{s} \\
& \times \frac{\left(b_{1}^{\dagger}\right)^{s+p}\left(b_{2}^{\dagger}\right)^{p+s-m}\left(b_{3}^{\dagger}\right)^{n_{0}+m-2 p-2 s}}{(2 p) ! !(2 l-m-2 p) !(2 m-2 l+2 p) ! !}|0,0,0\rangle,
\end{aligned}
$$

with $C_{n l m}$ given by (C.12) and $b_{i}^{\dagger}$ by (C.2).

\section{References}

[1] Jaynes E T and Cummings F W 1963 Proc. IEEE 5189

[2] See for instance Raimond J M et al 2001 Rev. Mod. Phys. 73565

[3] Ghosh P K 1995 Ion Traps (Oxford: Clarendon)

Major F G, Gheorghe V N and Werth G 2005 Charged Particle Traps (Berlin: Springer)

[4] Leibfried D et al 2003 Quantum dynamics of single trapped ions Rev. Mod. Phys. 75 281-324

Wineland D J, Monroe C, Itano W M, Leibfried D, King B E and Meekhof D M 1998 J. Res. Natl. Inst. Stand. Technol. 103259

[5] Vogel W and Wallentowitz S 2001 Manipulation of the quantum state of a trapped ion Coherence and Statistics of Photons and Atoms ed Jan Perina (New York: Wiley)

[6] Monroe C et al 1996 Science 2721131

Leibfried D et al 2005 Nature 438639

[7] Schmidt-Kaler F et al 2003 Nature $\mathbf{4 2 2} 408$

[8] Riebe M et al 2004 Nature 429734 Barrett M D et al 2004 Nature $\mathbf{4 2 9} 737$

[9] See for instance de Matos R L and Vogel W 1998 Phys. Rev. A 58 R1661 Vogel W and de Matos Filho R L 1995 Phys. Rev. A 524214

[10] Maniscalco S et al 2000 J. Mod. Opt. 472113 Maniscalco S et al 2001 J. Mod. Opt. 482065 Maniscalco S et al 2000 Phys. Rev. A 61053806

[11] Napoli A and Messina A 1997 Quantum Semiclass. Opt. 9587

[12] Greenberger D M, Horne M A, Shimony A and Zeilinger A 1990 Bell's theorem without inequalities Am. J. Phys. 58 1131-43

Hardy L 1998 Spooky action at a distance in quantum mechanics Contemp. Phys. 39 419-29

[13] Toschek P E 1984 Atomic particles in traps New Trends in Atomic Physics ed G Grynberg and R Stora (Amsterdam: Elsevier) p 282

[14] Nikitin A G 1998 On exact Foldy-Wouthuysen transformation J. Phys. A: Math. Gen. 31 3297-300

[15] Tsai W and Yildiz A 1971 Motion of charged particles in a homogeneous magnetic field Phys. Rev. D 4 3643-8

[16] Horvath G Z K et al 1997 Contemp. Phys. 3825

[17] Cooper F, Khare A and Sukhatme U 2001 Supersymmetry in Quantum Mechanics (Singapore: World Scientific)

[18] Moreno M, Martinez R and Zentella A 1990 Hidden supersymmetry of the Dirac equation and stability of the Dirac sea Mod. Phys. Lett. 5949

[19] Moshinsky M and Smirnov Yu M 1996 The Harmonic Oscillator in Modern Physics (The Netherlands: Harwood Academic) pp 2, 7

[20] Cohen-Tannoudji C, Diu B and Laloe F 1998 Quantum Mechanics (New York: Wiley-Interscience)

[21] Brihaye Y and Nininahazwe A 2005 Extended Jaynes-Cummings models and (quasi)-exact solvability Preprint quant-ph/0506249

[22] Van der Jeugt J 1993 Dynamical algebra of the $q$-deformed three-dimensional oscillator J. Math. Phys. 34 1799-806

Raychev P P et al 1996 Simplified boson realization of the $s o_{q}(3)$ subalgebra of $u_{q}(3)$ and matrix elements of

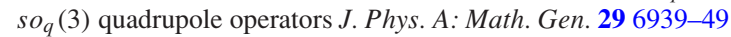

[23] Scala M, Militello B and Messina A 2006 Anisotropy-induced effects in the dynamics of an ion confined in a two-dimensional Paul trap Opt. Syst. Inform. Dyn. 13 315-21 